Supporting Information for

\title{
Enhanced Activation Energy Released by Coordination of Bifunctional Lewis Base D- Tryptophan for Highly Efficient and Stable Perovskite Solar Cells
}

Hanyu Wang*, Yukun Ouyang, Wenjing Zou, Xingchong Liu, Haimin Li, Ruonan Zhou, Xian Peng, Xiaoli Gong

School of New Energy and Materials, Southwest Petroleum University, Chengdu, 610500, China

*Corresponding authors. Email address: hywang@,swpu.edu.cn 
(a)

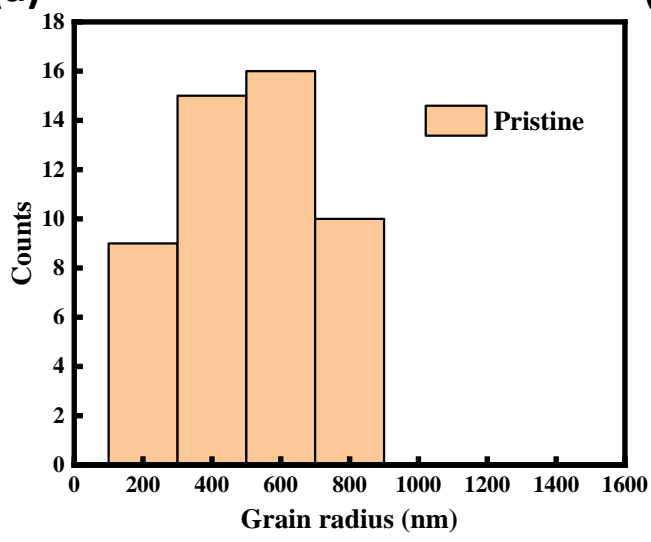

(b)

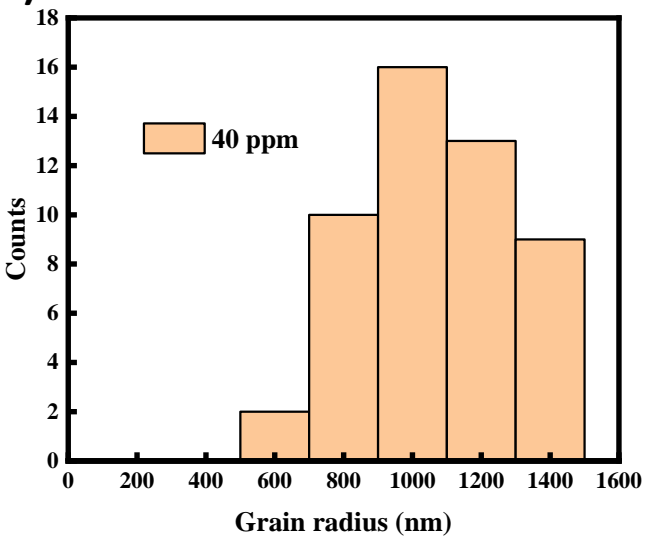

Figure S1. (a) Grain radius size distribution of pristine films, (b) grain radius size distribution of D-tryptophan-perovskite films. 


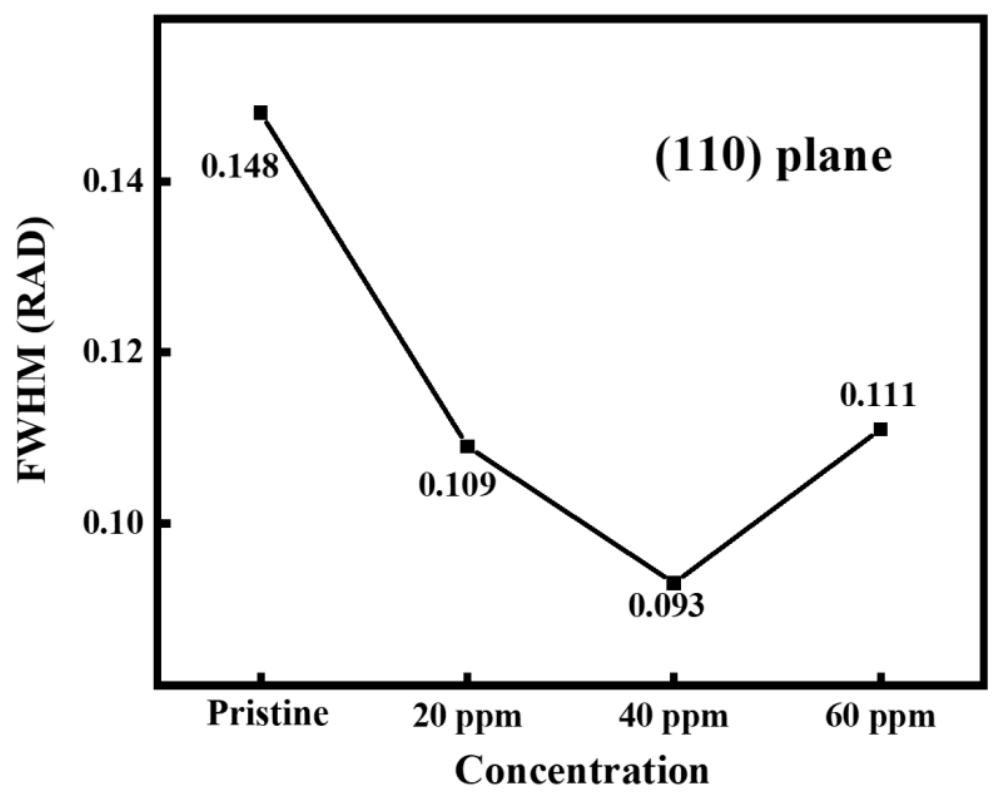

Figure S2. The FWHM of perovskite (110) plane with different D-tryptophan doping concentration. 
(a)

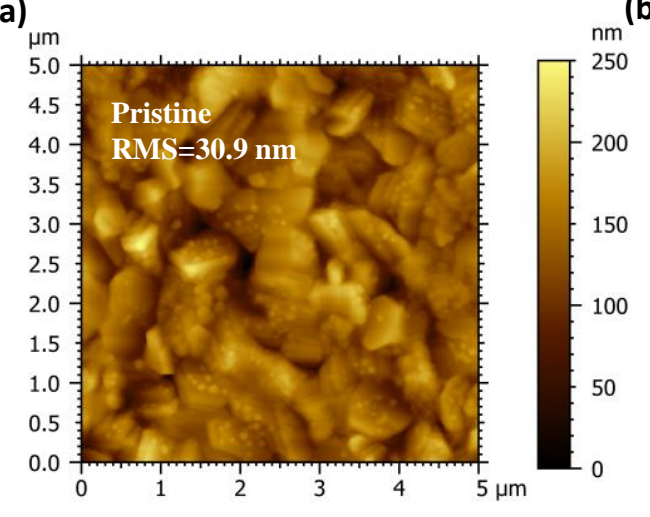

(b)

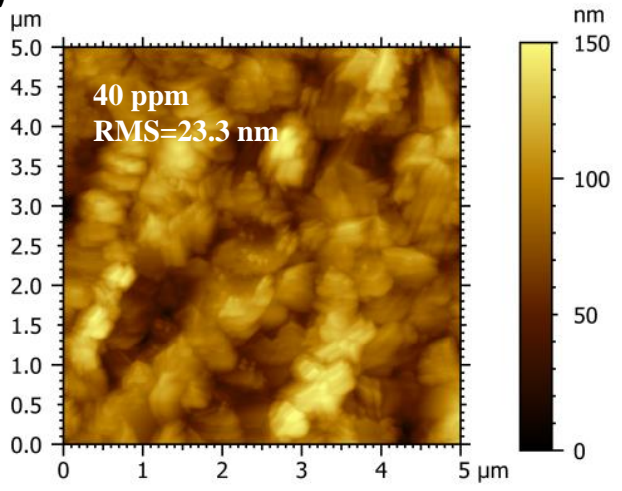

Figure S3. (a) AFM of pristine films, (b) AFM of D-tryptophan-perovskite films, with root mean square of surface roughness (RMS). 
(a)

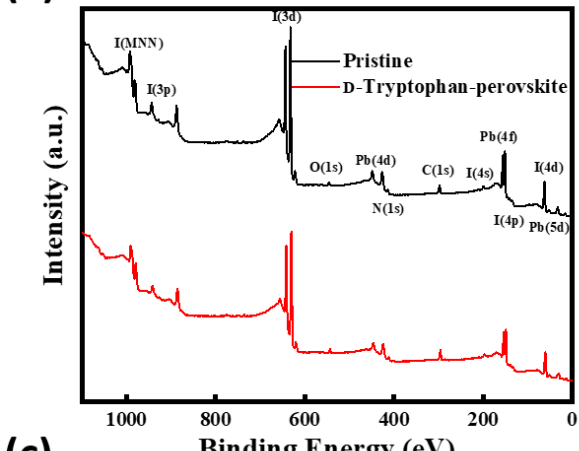

(c)

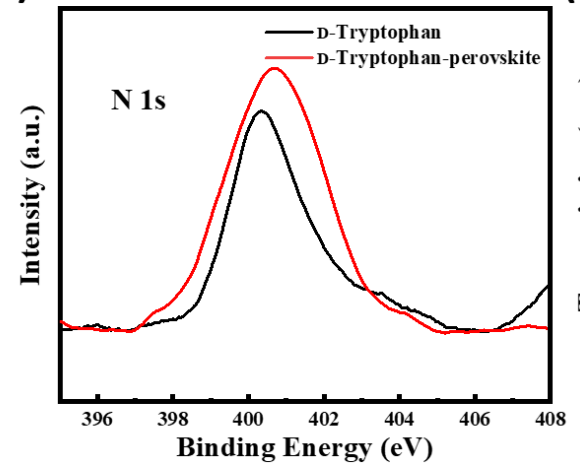

(b)

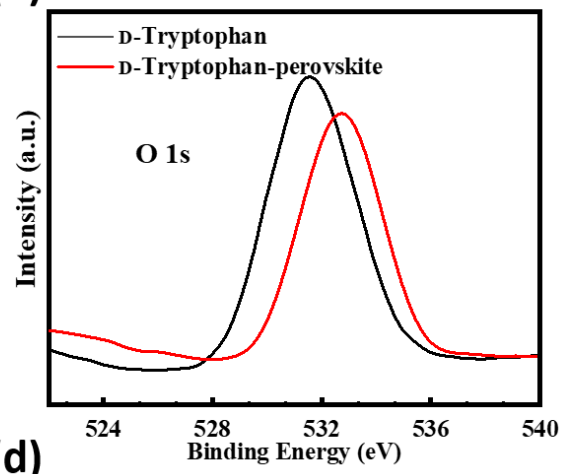

(d)

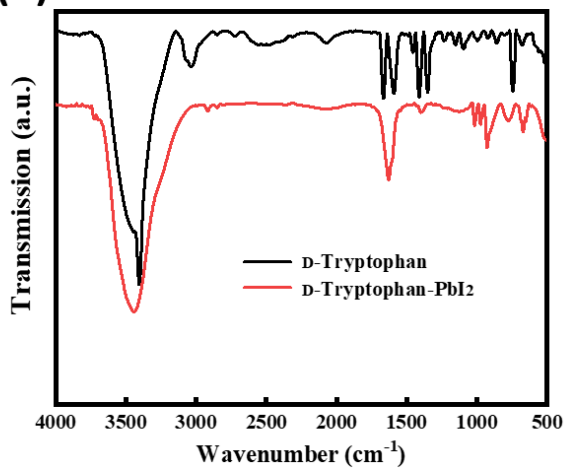

Figure S4. (a) XPS full spectrum of pristine and D-tryptophan-perovskite films, (b)XPS spectrum O 1s of D-tryptophan and D-tryptophan-perovskite films, (c) XPS spectrum N 1s of D-tryptophan and D-tryptophan-perovskite films. (d) FTIR spectra of D-tryptophan and Dtryptophan- $\mathrm{PbI}_{2}$. 
(a)

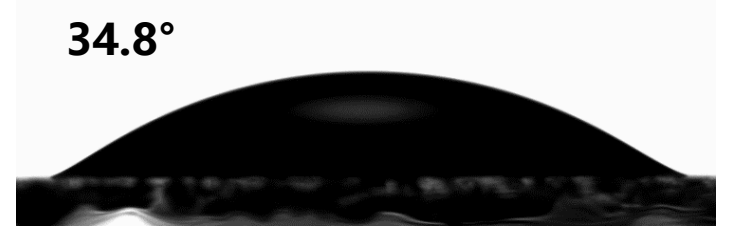

(b)

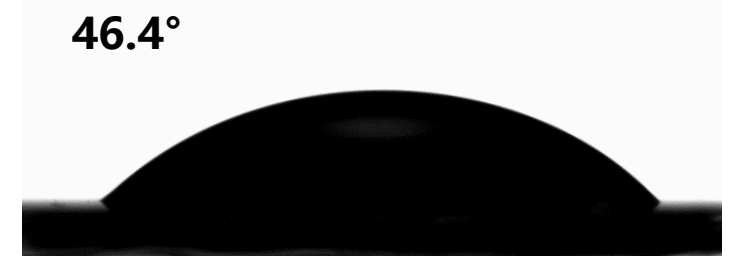

Figure S5. (a) Water contact angles on perovskite films without D-tryptophan, (b) Water contact angles on perovskite films with D-tryptophan 


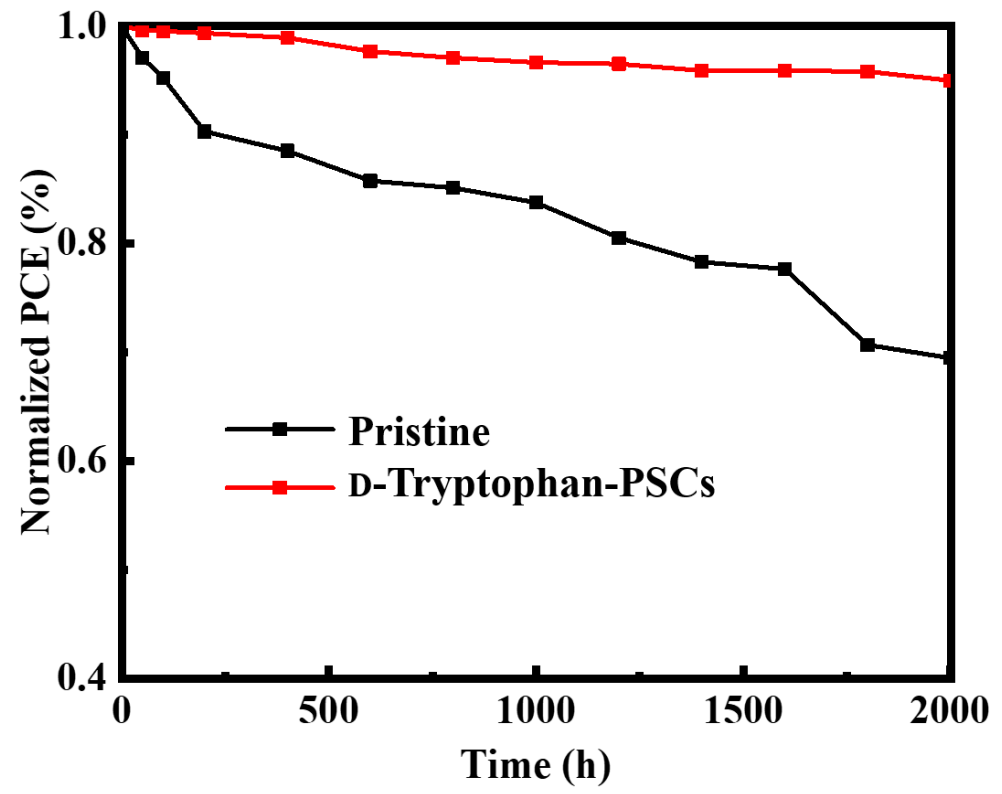

Figure S6. Stability of pristine and modified devices under $\mathrm{N}_{2}$ condition, at room temperature for 2000 hours. 
Table S1. TRPL data for perovskite films of pristine device and D-tryptophan-PSCs on glass substrate.

\begin{tabular}{cccccc}
\hline Sample & $\tau_{\text {ave }}(\mathrm{ns})$ & $\tau_{1}(\mathrm{~ns})$ & $A_{1}(\%)$ & $\tau_{2}(\mathrm{~ns})$ & $A_{2}(\%)$ \\
\hline Pristine & 202.81 & 36.58 & 2.19 & 203.47 & 97.81 \\
D-Tryptophan-PSCs & 634.82 & 78.51 & 2.36 & 636.48 & 97.64 \\
\hline
\end{tabular}

